\section{Inflammatory molecule reduction with hydroxyurea therapy in children with sickle cell anemia}

Children with sickle cell anemia (SCA) suffer from a chronic state of inflammation due to repeated tissue insult from sickling red blood cells. The clinical consequences are severe and involve aberration of inflammatory systems, with associated increased activation of multiple cell types, including platelets, neutrophils, and eosinophils. ${ }^{1,2}$ The literature has described a reduction of inflammatory molecules by hydroxyurea, but results have been limited, and at times contradictory. ${ }^{3-8}$ In fact, some researchers reported that patients receiving hydroxyurea exhibited increased levels of interleukin (IL)- 6 compared to untreated patients, ${ }^{9}$ while others reported a decrease in IL-6 levels in patients after initiating hydroxyurea therapy, ${ }^{10}$ albeit neither finding reached statistical significance (perhaps due to small study numbers). Herein, we address discrepancies by reporting a comprehensive study in which 214 patients with SCA were assessed, 122 of whom were sampled longitudinally. We compared patients on hydroxyurea (both initial and sustained therapy) with patients on chronic blood transfusions or no treatment. In grouped analyses, hydroxyurea proved to be the better treatment for reduction of inflammatory molecules. During the 2-year observation period, children continued to benefit from hydroxyurea treatment, although they did not achieve profiles similar to those of healthy controls. Patients receiving continuous chronic blood transfusion therapy or no treatment showed fewer reductions in factor levels compared to hydroxyurea. In total, our data illustrated the long-term, but incomplete benefit of hydroxyurea for reduction of inflammatory molecules in patients with SCA.

The large population of children with SCA at St. Jude Children's Research Hospital provided us with an opportunity to conduct a comprehensive and longitudinal study of cytokines, chemokines, and adhesion molecules in the context of hydroxyurea treatment, chronic transfusions, or no treatment. Our study was initiated by examining 41 serum factors among 214 children with SCA and
15 healthy controls (see Methods in Online Supplementary Materials). Ninety-two of the 214 patients were sampled only once during the study ('baseline') whereas 122 patients were sampled longitudinally, before and after a 2 -year observation period ('baseline' and 'follow-up'). Patient genotypes were $\mathrm{HbSS}(\mathrm{N}=200)$ and $\mathrm{HbS \beta} 0$ thalassemia $(\mathrm{N}=14)$. The median age of patients at baseline was 10.4 years (range: 5.2 to 18.8 years); 105 patients were male; 109 patients were female; all patients were African American. The SCA subgroups, according to treatment status at baseline assessment, were as follows: untreated $(\mathrm{N}=118)$, treated with hydroxyurea at maximum tolerated dose (MTD, N=72), or treated with chronic monthly erythrocyte transfusions $(\mathrm{N}=24)$. Among the 122 patients followed longitudinally for two years, 26 were initially untreated and subsequently started on hydroxyurea treatment at some point during the 2year observation period ('initiating hydroxyurea'), 41 had previously initiated treatment and were continuously on hydroxyurea therapy ('continuous hydroxyurea') for two additional years, 17 children were on continuous blood transfusion therapy, and 38 children never received any disease-modifying therapy (Online Supplementary Table S1). Plasma from 15 healthy controls (median age 13.0 years, range: $6.5-19.0$ years) was evaluated for the same inflammatory molecules. Fourteen of these children were African American and one was Caucasian. Their sex distribution was three males and 12 females. It should be noted that previous studies have shown that a majority of the factors evaluated in this study are equally detectable in plasma and sera, showing no significant difference in detectability. ${ }^{11}$ Factors that may be affected by blood source include endothelial growth factor (EGF), macrophage inflammatory protein $1 \beta$ (MIP-1 $\beta$ ) and soluble CD40-ligand (sCD40L), which may be underrepresented 1-3-fold in plasma, as well as inducible protein 10 (IP-10) and MIP-1 $\alpha$, which may be overrepresented 1.53 -fold in plasma.

When all children with SCA were sampled as a single group at baseline $(n=214)$, they exhibited higher levels of inflammatory markers than children without SCA. Of the 41 factors evaluated, 16 were found to have elevated

Table 1. Correlation of changes in cytokines with changes in clinical inflammatory markers.

\begin{tabular}{|c|c|c|c|c|c|c|c|c|c|}
\hline \multirow{3}{*}{ Change in cytokines } & \multicolumn{7}{|c|}{ Continuous hydroxyurea therapy } & & \\
\hline & \multicolumn{3}{|c|}{ Change in Platelet Count } & \multicolumn{3}{|c|}{ Change in wWFAg } & \multicolumn{3}{|c|}{ Change in WBC } \\
\hline & N & rs & $P$ & $\mathbf{N}$ & is & $\mathbf{P}$ & N & rs & P \\
\hline VCAM-1 & 41 & 0.25 & 0.115 & 40 & 0.35 & 0.025 & 41 & 0.15 & 0.351 \\
\hline VEGF & 41 & 0.35 & 0.023 & 40 & 0.15 & 0.365 & 41 & 0.30 & 0.060 \\
\hline E-selectin & 41 & 0.22 & 0.177 & 40 & 0.38 & 0.016 & 41 & 0.59 & $<.001$ \\
\hline sCD40L & 41 & -0.02 & 0.924 & 40 & 0.17 & 0.287 & 41 & 0.02 & 0.921 \\
\hline \multirow[t]{2}{*}{ Change in cytokines } & \multicolumn{6}{|c|}{$\begin{array}{l}\text { Initiated hydroxyurea therapy } \\
\text { Change in Platelet Count } \\
\text { Change in wWF: }\end{array}$} & \multicolumn{3}{|c|}{ Change in WBC } \\
\hline & N & rs & P & N & rs & P & N & rs & $\mathbf{P}$ \\
\hline VCAM-1 & 26 & 0.02 & 0.922 & 25 & 0.44 & 0.027 & 26 & 0.42 & 0.034 \\
\hline VEGF & 26 & -0.33 & 0.096 & 25 & 0.06 & 0.770 & 26 & 0.06 & 0.770 \\
\hline E-selectin & 26 & 0.30 & 0.133 & 25 & 0.34 & 0.093 & 26 & 0.56 & 0.003 \\
\hline sCD40L & 26 & -0.005 & 0.982 & 25 & -0.12 & 0.583 & 26 & 0.03 & 0.894 \\
\hline
\end{tabular}

$\mathrm{N}$ : number of patients; rs: Spearman rank correlation coefficient; $P$ : value for the correlation coefficient. vWF:Ag: von Willebrand antigen level; WBC: white blood cell count; VCAM-1: vascular adhesion molecule 1;VEGF: vascular endothelial growth factor; sCD40L: soluble CD40-ligand. 

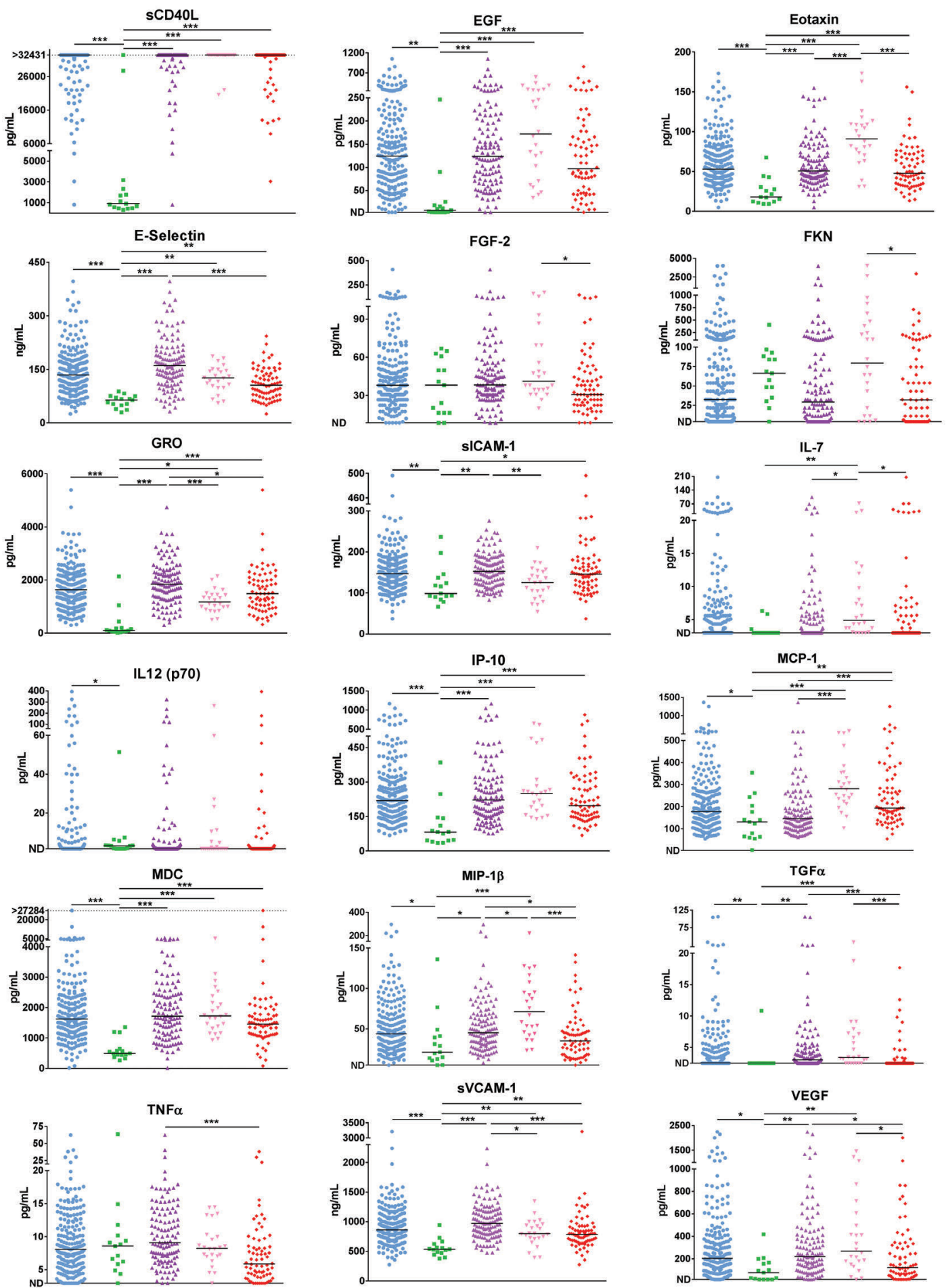

\section{All SCA \\ Healthy Child \\ SCA-No Treatment \\ SCA-Chronic Transfusions}

SCA-Hydroxyurea

Figure 1. Grouped analyses of healthy children and children with sickle cell anemia (SCA) on various treatments. Groups of children were monitored for serum cytokines, chemokines and adhesion molecules using the Luminex platform and by enzyme-linked immunosorbent assay (ELISA). Each child is represented only once within a group. All factors for which there were any significant differences between any of the SCA patient groups (healthy controls, SCA-No Treatment, SCA-Hydroxyurea, SCA-Chronic Transfusions) are shown. Differences between patients with SCA and healthy controls were determined using the Mann-Whitney $\mathrm{U}$ test. Significance amongst treatment groups were evaluated using a Kruskal-Wallis test followed by a Dunn's pairwise test. $P$-values were adjusted for multiple testing across inflammatory molecules with the Benjamini-Hochberg false discovery rate (FDR) method (please see Online Supplementary Table S3 and Online Supplementary Table S5 for exact and FDR-corrected $P$-values). $* P \leq 0.05, * * P \leq 0.01, * * * P \leq 0.001$. EGF: endothelial growth factor; MIP-1 $\beta$ : macrophage inflammatory protein 1 $\beta$; sCD40L: soluble CD40-ligand; IL: interleukin; IP-10: inducible protein 10; GRO: growth-related oncogene; TGF $\alpha$ : transforming growth factor $\alpha$; TNF $\alpha$ : tumor necrosis factor $\alpha$; sVCAM-1: soluble vascular adhesion molecule 1; VEGF: vascular endothelial growth factor; MCP-1: monocyte chemoattractant protein 1; FGF-2: fibroblast growth factor 2; sICAM-1: soluble intercellular adhesion molecule 1; MDC: macrophage-derived chemokine; FKN: fracktalkine. 
median values (12 of which were significant) in children with SCA compared to healthy controls (Online Supplementary Table S2 and Online Supplementary Table S3). Only IL-12p70 showed a significantly decreased median in SCA patients compared to healthy controls. Known functions and SCA-related literature for each significantly different factor can be found in Online
Supplementary Table S4. When mean values were compared between untreated children with SCA and healthy controls, 34 of the 41 factors were elevated.

At baseline assessment, the median duration of hydroxyurea treatment was 4.7 years and the median duration of transfusion therapy was 6.3 years (see Online Supplementary Methods). When children receiving hydrox-
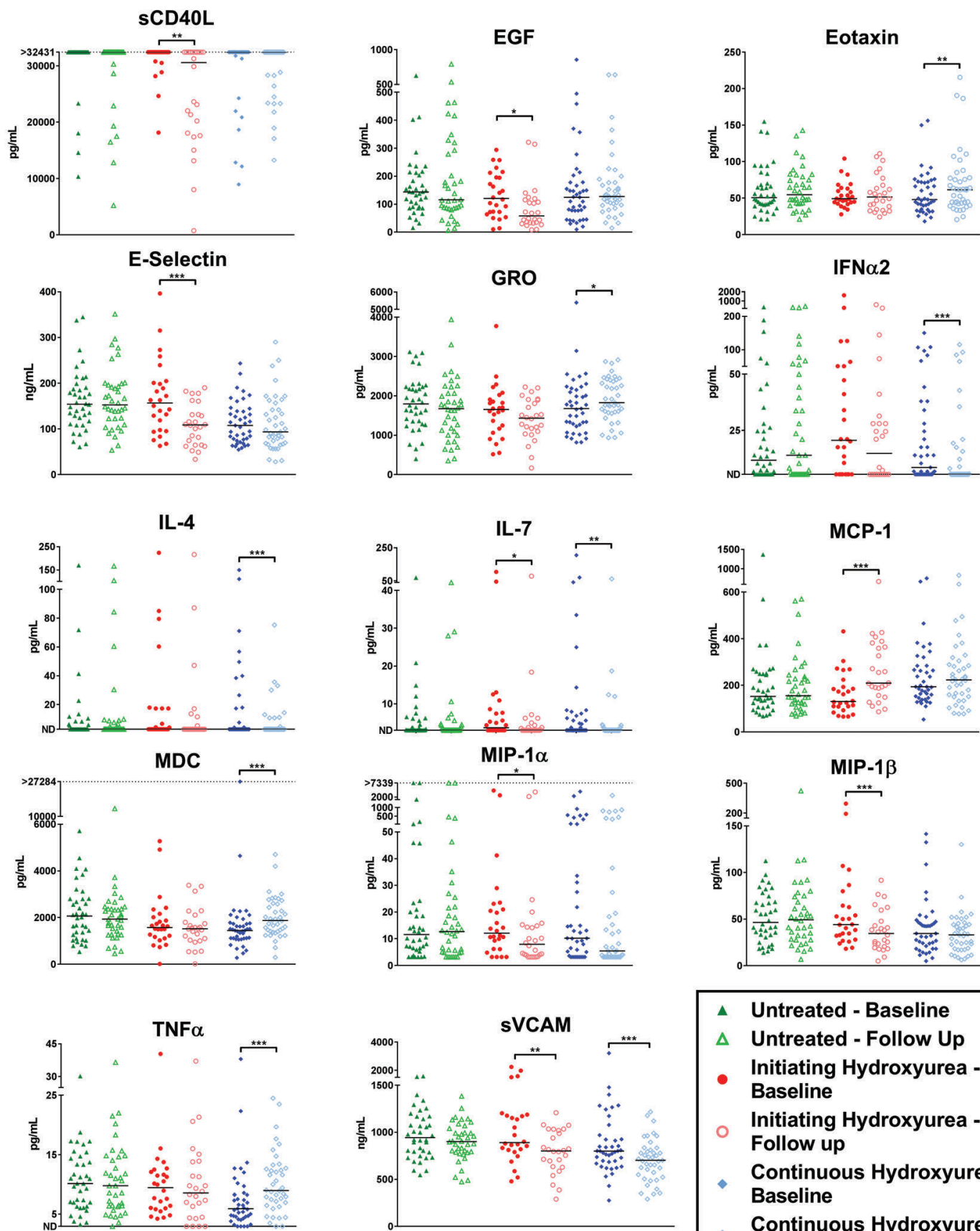
$\Delta \quad$ Untreated - Baseline
$\Delta$ Untreated - Follow Up
Initiating Hydroxyurea -
- Baseline
Initiating Hydroxyurea -
Follow up
Continuous Hydroxyurea -
Baseline
Continuous Hydroxyurea -
Follow Up

Figure 2. Analyses of children with SCA who switched from no treatment to hydroxyurea therapy during a 2-year observation period. Children were monitored for serum cytokines, chemokines and adhesion molecules before and after a 2-year period during which they: (i) received no treatment, (ii) were switched from no treatment to hydroxyurea, or (ii) continuously received hydroxyurea. Significant changes from baseline to follow-up sampling were determined using the Wilcoxon signed-rank test. $* P \leq 0.005, * * P \leq 0.01, * * * P \leq 0.001$. EGF: endothelial growth factor; MIP-1 $\alpha$ : macrophage inflammatory protein $1 \alpha$; sCD40L: soluble CD40-ligand; IL: interleukin; IP-10: inducible protein 10; GRO: growth-related oncogene; TGF $\alpha$ : transforming growth factor $\alpha$; TNF $\alpha$ : tumor necrosis factor $\alpha$; sVCAM: soluble vascular adhesion molecule; MCP-1: monocyte chemoattractant protein 1; FGF-2: fibroblast growth factor 2; sICAM-1: soluble intercellular adhesion molecule 1; IFN $\alpha 2$ : interferon $\alpha 2$; MDC: macrophage-derived chemokine. 
yurea therapy at baseline were compared to untreated children with SCA, significant differences were noted (Figure 1, Online Supplementary Table S2 and Online Supplementary Table S5) including reductions in soluble (s)E-selectin, growth-related oncogene (GRO), MIP-1 $\beta$, transforming growth factor $\alpha$ (TGF $\alpha)$, tumor necrosis factor $\alpha(T N F \alpha)$, soluble vascular adhesion molecule 1 (sVCAM-1) and vascular endothelial growth factor (VEGF). Only one factor, monocyte chemoattractant protein 1 (MCP-1), was significantly elevated in hydroxyurea-treated children. Even though levels of immune and adhesion molecules were generally reduced in hydroxyurea-treated patients, they often remained higher than those of healthy controls. When patients with SCA receiving chronic blood transfusions at baseline were evaluated, (Figure 1 and Online Supplementary Table S2), some factors were significantly reduced compared to untreated patients, but most measurements remained higher than those for patients treated with hydroxyurea.

Twenty-six untreated children initiated hydroxyurea between baseline sampling and the 2 -year assessment date (median duration of treatment was 1.3 years). This group afforded us the unique opportunity to evaluate the early influence of hydroxyurea on immune and adhesion molecules at an individual patient level. Each patient served as its own control, allowing person-to-person variability to be avoided. Initiation of hydroxyurea treatment associated with a decrease in 19 of the factors examined (Online Supplementary Table S6A), seven of which were significantly decreased, including sCD40L, EGF, sE-selectin, IL-7, MIP- $1 \alpha$, MIP- $1 \beta$, and sVCAM-1 (Figure 2). It is important to note that no significant differences were found in patients who remained untreated throughout the 2-year interval $(\mathrm{N}=38)$, even though factors such as sE-selectin and sVCAM-1 were expected to decrease with age in non-SCA individuals ${ }^{12}$ (Figure 2 and Online Supplementary Table S6B).

Forty-one children were treated continuously with hydroxyurea during the 2 -year observation period (median duration of therapy at baseline sampling was 4.8 years). In this cohort, interferon $\alpha 2$ (IFN $\alpha 2$ ) and sVCAM1 were found to be reduced significantly over the 2-year interval (Figure 2 and Online Supplementary Table S6A). Additionally, although the median values were equivalent for IL-4 and IL-7, the mean values and the number of patients with elevated levels (above the limit of detection) were significantly reduced. These results suggest that long-term treatment with hydroxyurea can sustain or further reduce inflammatory markers. This benefit was not observed in the participants who received transfusions. Of the 17 chronic transfusion patients evaluated, no significant decreases in factor levels over time were observed over the 2-year observation period, though TNF $\alpha$ was found to increase significantly (Online Supplementary Table S6B). This increase may simply be a reflection of the fact that TNF $\alpha$ is known to increase with age ${ }^{13}$ this increase is not observed in newly hydroxyureatreated (initiating hydroxyurea) patients, likely due to the anti-inflammatory effects of the treatment (Figure 2).

Finally, we examined the longitudinal change of six inflammatory markers: white blood cell count (WBC), platelet count, von Willebrand antigen level (vWF:Ag), Creactive protein, D-dimer, and Factor VIII antigen level. Only in the initiated hydroxyurea group were WBC, platelet count, and vWF antigen levels significantly reduced (Online Supplementary Table S7). The remaining inflammatory markers did not significantly change in any of the other SCA groups. The decline in WBC, platelet count, and vWF during hydroxyurea was significantly associated with reductions in VCAM-1, VEGF, and E-selectin (Table 1). Paralleling the cytokine and inflammatory marker reduction, the incidence of vaso-occlusive events (VOEs) reduced in both the continuous and initiated hydroxyurea groups: 15.9 to $4.9 / 100$ patients-years $(P=0.01)$, and 21.2 to $5.8 / 100$ patient-years $(P=0.009)$, respectively. No significant changes in VOEs were observed in the continuous transfusions or untreated groups (Online Supplementary Table S8).

Collectively, when expressed at high levels in patients with SCA, immune and adhesion molecules indicate a hyperinflammatory state due to widespread tissue damage, a likely consequence of vaso-occlusion and tissue ischemia. ${ }^{14}$ Activated cells that contribute to elevated serum factors include endothelial cells (expressing selectins and adhesion molecules), monocytes (expressing TNF $\alpha$ and IL1 $\beta$ ), and T-cells (expressing interleukins). The precise array of factors in each patient sample was likely dependent on numerous variables, including the frequency, magnitude, and location of tissue damage. The reduction in inflammatory factors described herein suggested that treatments (particularly hydroxyurea) reduced damage, perhaps by increasing fetal hemoglobin and conferring mild myelosuppression.

Our results improve upon those of previous studies in that we were able to monitor a large number of children with SCA longitudinally over a 2 -year period. Some reductions with hydroxyurea treatment matched previously described results, such as reductions in TNF- $\alpha$, VEGF and E-selectin, ${ }^{5,15,16}$ but others were new, for instance reductions in sCD40L, GRO and MIP-1 $\beta$. Overall, we demonstrate that inflammatory markers are elevated in children with SCA in comparison with healthy controls, and also higher among untreated children in relationship with those exposed to disease-modifying therapies. Hydroxyurea therapy reduced inflammatory molecule levels more efficiently and its prolonged use sustained the reduction of most of these factors at lower levels, and paralleled a reduction in VOEs and clinical inflammatory markers, whereas chronic transfusion therapy did not reduce inflammatory molecules to the same degree.

As hydroxyurea treatments improved, but did not fully correct inflammation in patients with SCA, advanced drug development is warranted. Several new drugs with specific anti-inflammatory targets are currently in development. Anti-adhesion agents such as anti-E selectin (rivipansel, formerly GMI-1070), ${ }^{17}$ anti-P selectin (crizanlizumab), ${ }^{18}$ and broad anti-adhesives (simvastatin, AKT inhibitors), ${ }^{19}$ used individually or in combination with hydroxyurea, have been shown to reduce VOEs. An opportunity now exists to further test new drugs in combination with hydroxyurea in order to potentiate the benefits provided by hydroxyurea therapy alone. The results herein may serve as a guide against which new anti-inflammatory therapies for SCA can be compared.

Rhiannon R. Penkert, ${ }^{1}$ Julia L. Hurwitz, ${ }^{1,2}$ Paul Thomas, ${ }^{3}$ Jason Rosch, Jola Dowdy, ${ }^{4}$ Yilun Sun, ${ }^{5}$ Li Tang ${ }^{5}$ and Jane S. Hankins $^{4}$

Departments of Infectious Diseases, ${ }^{3}$ Immunology, ${ }^{4}$ Hematology, and 'Biostatistics, St. Jude Children's Research Hospital and ${ }^{2}$ Department of Microbiology, Immunology and Biochemistry, University of Tennessee Health Science Center, Memphis, TN, USA

Acknowledgments: the authors would like to thank Teresa Carr, $R N, B S$ for support with regulatory matters, and Russell Ware, MD, PhD, Amber Yates, MD, Alyssa Cotton, FNP and Paul Lavoie, MHS, PA-C, for support with patient enrollment. 
Funding: this work was supported by NCI P30 CA21765, a grant from the Children's Infection Defense Center, and ALSAC.

Correspondence: jane.hankins@stjude.org

doi:10.3324/haematol.2017.177360

Information on authorship, contributions, and financial \& other disclosures was provided by the authors and is available with the online version of this article at www. haematologica.org.

\section{References}

1. Canalli AA, Conran N, Fattori A, et al. Increased adhesive properties of eosinophils in sickle cell disease. Exp Hematol. 2004;32(8):728734.

2. Anyaegbu CC, Okpala IE, Akren'Ova YA, et al. Peripheral blood neutrophil count and candidacidal activity correlate with the clinical severity of sickle cell anaemia (SCA). Eur J Haematol. 1998;60(4):267268.

3. Johnson C, Telen MJ. Adhesion molecules and hydroxyurea in the pathophysiology of sickle cell disease. Haematologica. 2008;93(4):481-485

4. Lapoumeroulie C, Benkerrou M, Odievre MH, et al. Decreased plasma endothelin-1 levels in children with sickle cell disease treated with hydroxyurea. Haematologica. 2005;90(3):401-403.

5. Lebensburger JD, Howard T, Hu Y, et al. Hydroxyurea therapy of a murine model of sickle cell anemia inhibits the progression of pneumococcal disease by down-modulating E-selectin. Blood. 2012;119(8):1915-1921.

6. Odievre MH, Bony V, Benkerrou M, et al. Modulation of erythroid adhesion receptor expression by hydroxyurea in children with sickle cell disease. Haematologica. 2008;93(4):502-510.

7. Sarray S, Saleh LR, Lisa Saldanha F, et al. Serum IL-6, IL-10, and TNFalpha levels in pediatric sickle cell disease patients during vasoocclusive crisis and steady state condition. Cytokine. 2015;72(1):43-47.

8. Styles LA, Lubin B, Vichinsky E, et al. Decrease of very late activation antigen-4 and CD36 on reticulocytes in sickle cell patients treated with hydroxyurea. Blood. 1997;89(7):2554-2559.

9. Keikhaei B, Mohseni AR, Norouzirad R, et al. Altered levels of proinflammatory cytokines in sickle cell disease patients during vasoocclusive crises and the steady state condition. Eur Cytokine Netw. 2013;24(1):45-52.

10. Saleh AW, Duits AJ, Gerbers A, de VC, Hillen HF. Cytokines and soluble adhesion molecules in sickle cell anemia patients during hydroxyurea therapy. Acta Haematol. 1998;100(1):26-31.

11. Alsaif M, Guest PC, Schwarz E, et al. Analysis of serum and plasma identifies differences in molecular coverage, measurement variability, and candidate biomarker selection. Proteomics Clin Appl. 2012;6(5-6):297-303

12. Nash MC, Wade AM, Shah V, Dillon MJ. Normal levels of soluble Eselectin, soluble intercellular adhesion molecule-1 (sICAM-1), and soluble vascular cell adhesion molecule-1 (sVCAM-1) decrease with age. Clin Exp Immunol. 1996;103(1):167-170.

13. Kleiner G, Marcuzzi A, Zanin V, et al. Cytokine levels in the serum of healthy subjects. Mediators Inflamm. 2013;2013:434010.

14. Pitanga TN, Vilas-Boas W, Cerqueira BAV, et al. Cytokine profiles in sickle cell anemia; Pathways to be unraveled. Adv Biosci Biotechnol. 2013:4:6-12.

15. Lanaro C, Franco-Penteado CF, Albuqueque DM, et al. Altered levels of cytokines and inflammatory mediators in plasma and leukocytes of sickle cell anemia patients and effects of hydroxyurea therapy. J Leukoc Biol. 2009;85(2):235-242.

16. Lopes FC, Traina F, Almeida CB, et al. Key endothelial cell angiogenic mechanisms are stimulated by the circulating milieu in sickle cell disease and attenuated by hydroxyurea. Haematologica. 2015;100(6):730-739.

17. Chang J, Patton JT, Sarkar A, et al. GMI-1070, a novel pan-selectin antagonist, reverses acute vascular occlusions in sickle cell mice Blood. 2010;116(10):1779-1786.

18. Ataga KI, Kutlar A, Kanter J, et al. Crizanlizumab for the prevention of pain crises in sickle cell disease. N Engl J Med. 2017;376(5):429439 .

19. Hoppe C, Jacob E, Styles L, et al. Simvastatin reduces vaso-occlusive pain in sickle cell anaemia: a pilot efficacy trial. Br J Haematol. 2017;177(4):620-629. 